\title{
Spectral calibration in hyperspectral sounders
}

Evan M. Manning, Hartmut H. Aumann, Robert G. Deen, Yibo Jiang, L. Larrabee Strow, et al.

Evan M. Manning, Hartmut H. Aumann, Robert G. Deen, Yibo Jiang, L. Larrabee Strow, Scott E. Hannon, "Spectral calibration in hyperspectral sounders," Proc. SPIE 7452, Earth Observing Systems XIV, 74520H (21 August 2009); doi: 10.1117/12.826987

SPIE Event: SPIE Optical Engineering + Applications, 2009, San Diego, California, United States 


\title{
Spectral calibration in hyperspectral sounders ${ }^{*}$
}

\author{
Evan M. Manning ${ }^{\mathrm{a}}$, Hartmut H. Aumann ${ }^{\mathrm{a}}$, Robert G. Deen ${ }^{\mathrm{a}}$, Yibo Jiang ${ }^{\mathrm{a}}$, L. Larrabee Strow ${ }^{\mathrm{b}}$, \\ and Scott E. Hannon ${ }^{\mathrm{b}}$ \\ ${ }^{a}$ Jet Propulsion Laboratory, California Institute of Technology, Pasadena, CA 91109 \\ ${ }^{\mathrm{b}}$ University of Maryland Baltimore County, Baltimore, MD
}

\begin{abstract}
It is widely accepted that the knowledge of the frequencies of the spectral response functions (SRF) of the channels of hyperspectral sounders at the 10 parts per million (ppm) of frequency level is adequate for the retrieval of temperature and moisture profiles and data assimilation for weather forecasting. However, SI traceability and knowledge at the $1 \mathrm{ppm}$ level and better are required to separate artifacts in the knowledge of the SRF due to orbital and seasonal instrument effects from diurnal and seasonal effects due to climate change. We use examples from AIRS to discuss a spectral calibration that uses the SI traceable upwelling radiance spectra to achieve an absolute accuracy of $0.5 \mathrm{ppm}$.
\end{abstract}

Keywords: Atmospheric Infrared Sounder AIRS Infrared Atmosphere Sounding Interferometer IASI Climate

\section{INTRODUCTION}

The spectrally resolved upwelling infrared radiation measured by hyperspectral sounders like the Atmospheric Infrared Sounder ${ }^{1}$ (AIRS), the Infrared Atmosphere Sounding Interferometer ${ }^{2}$ (IASI), and the Crosstalk Infrared Sounder ${ }^{3}$ (CrIS) have the potential to give unprecedented insight into the state of the atmosphere and to observe changes due to climate. The effect of climate changes will be as subtle as the $100 \mathrm{mK} /$ decade rate of global warming accepted in the 2007 IPCC report and even smaller changes are expected in day/night differences of surface and atmospheric temperatures. Use of hyperspectral data for climate is therefore extremely demanding on the absolute accuracy and stability of the radiometric and spectral calibration. In this paper we discuss the challenge posed by spectral shifts and present the new approach to the problem embodied in the upcoming v6 release of AIRS products.

\subsection{The challenge of spectral calibration}

If the absolute position of the spectral response function (SRF) is not precisely known, the difference between the actual position of the SRF in the frequency domain at the time of the observation and the assumed (nominal) position of the SRF acts as a source of radiometric error. We discuss this frequency uncertainty in units of parts per

\section{Radiometric change with $8 \mathrm{ppm}$ frequency spectral shift}

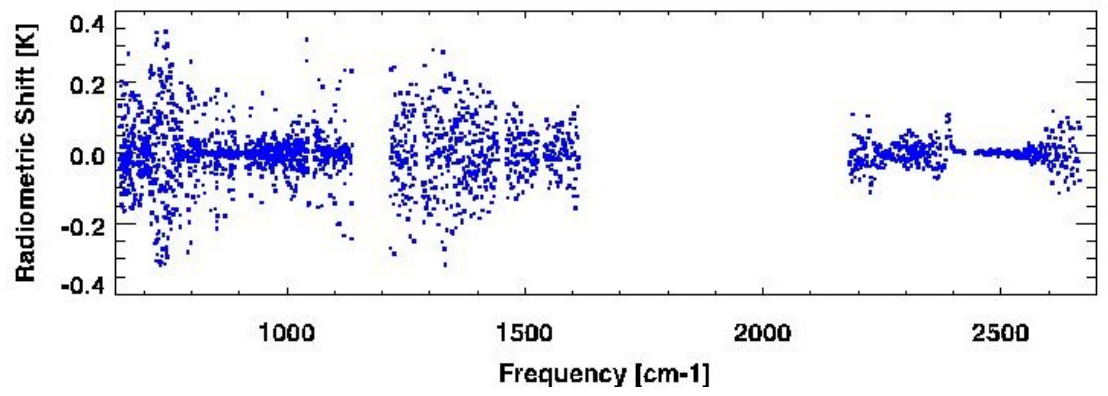

Figure 1. Radiometric change in a simulated AIRS clear tropical ocean spectrum for a frequency shift of $8 \mathrm{ppm}$.

\footnotetext{
* SPIE Optics-Photonics Meeting 2-6 August 2009, San Diego, California. Opinions, interpretations, conclusions, and recommendations are those of the authors and not necessarily endorsed by the United States Government.
} 
million (ppm) in frequency. The AIRS functional requirements stipulated that the frequency must be known and stable to within 8 ppm in any 24 hour period, but did not specify changes over a longer period. Figure 1 shows the radiometric effect of shifting a clear tropical AIRS ocean spectrum by $8 \mathrm{ppm}$. The mean over all channels is essentially zero, but the standard deviation is $0.09 \mathrm{~K}$, with some channels in particularly steep areas of the spectrum shifting by nearly $0.4 \mathrm{~K}$ for an 8 ppm shift in the SRF frequency.

The scatter of Figure 1 obscures the fact that the great majority of channels have very little sensitivity to frequency shift. Figures 2 and 3 show logarithmic histograms of the bias and RMS over one typical AIRS granule shifted by 8 ppm in frequency. Figure 3 shows that less than $1 \%$ of the channels change by more than $15 \mathrm{mK}$ for a frequency shift of $8 \mathrm{ppm}$. This is due to the fact that a large fraction of the channels are atmospheric window channels. The atmosphere sounding channels are typically in areas of much higher spectral contrast.

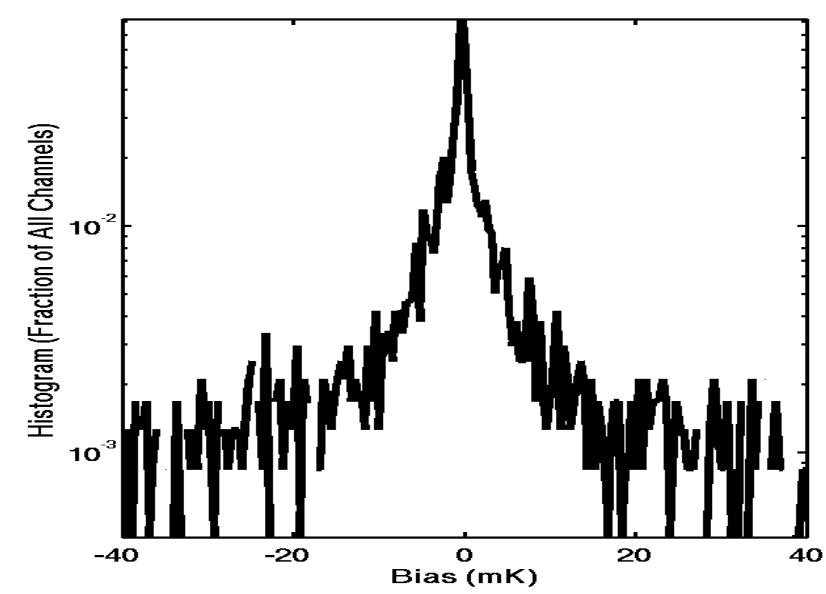

Figure 2. Histogram over one 6-minute granule of average bias resulting from an 8 ppm uncorrected spectral shift for AIRS.

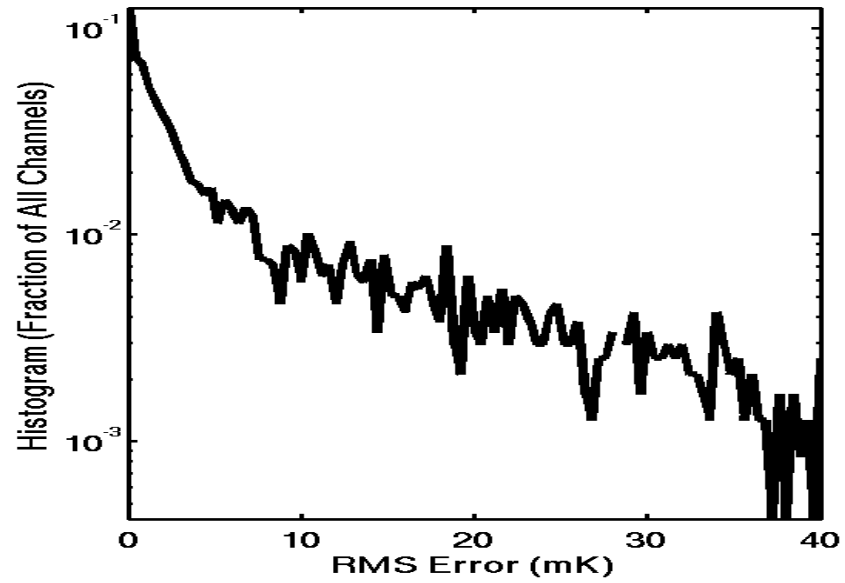

Figure 3. Histogram over one 6-minute granule of RMS error resulting from an 8 ppm uncorrected spectral shift for AIRS.

We illustrate one consequence of spectral calibration uncertainty with an example from IASI and AIRS using the 712.75 $\mathrm{cm}^{-1}$ channel, which sounds the atmosphere near $400 \mathrm{hPa}$, where the temperature in a tropical atmosphere is about $250 \mathrm{~K}$. The day/night temperature difference at this level can be used as proxy for the strength of convection, a key metric of climate change. Based on the analysis of two years of data the mean temperature in the non-frozen oceans from IASI at 9:30 AM (morning) overpasses is $180 \mathrm{mK}$ warmer than at the 9:30 PM overpasses, while for AIRS the temperature at the 1:30 PM (early afternoon) overpasses is $240 \mathrm{mK}$ warmer than during the 1:30 AM overpasses. The $60 \mathrm{mK}$ difference between the two measurements looks like an interesting climate signal, but first we must exclude instrument effects. A $60 \mathrm{mK}$ temperature difference corresponds to a change of only $\sim 3 \mathrm{ppm}$ in the frequency at $612.25 \mathrm{~cm}^{-1}$. If the frequency uncertainty were reduced to $0.5 \mathrm{ppm}$, its radiometric effect would contribute only $10 \mathrm{mK}$ to observed $60 \mathrm{mK}$ day/night shift and produce a measurement of value for climate. Based on similar analysis of other tropospheric channels we conclude that an absolute spectral calibration accuracy of $0.5 \mathrm{ppm}$ is required on a time scale that is a small fraction of the orbital period of 100 minutes in order to enable a secure interpretation of day/night climate-related effects.

\subsection{The origin of spectral shifts in orbit}

The sun-synchronous polar orbits employed by hyperspectral sounders present a challenging thermal environment. The primary driver is the heating and cooling of the spacecraft as it spends roughly half of each orbit in Earth's shadow. There are also more subtle modulations of temperatures from seasonal and weather-related changes in upwelling radiation from Earth, which warms the spacecraft and instruments from below, and from sunlight hitting different parts of the spacecraft at different points in the sunny part of the orbit.

In the case of a Fourier Transform Spectrometer (FTS), like IASI or CrIS, it is frequently assumed that the stability of the frequency of the metrology laser insured frequency accuracy and stability of the spectral channels. This is not the case, since orbital temperature variation cause misalignments in either the metrology laser relative to the optical bench or 
the focal plane relative to the FTS optical axis, causing the metrology laser derived frequencies to be in error. This is the main reason that IASI data are distributed only after an explicit frequency calibration using the upwelling spectra as a Level-1C product.

In the case of AIRS, which is a grating array spectrometer, day/night orbital temperature changes in the spacecraft frame, changes in the solar illumination and longer term mechanical relaxation effects cause shifts in $\mathrm{y}_{0}$, the position of the focal plane relative to the optical axis, of the order of 0.5 microns peak-to-peak. The AIRS spectrometer exit slit image in the focal plane is $100 \mu \mathrm{m}$ wide, so a $0.5 \mu \mathrm{m}$ shift results in a $0.5 \%$ shift in the SRF centroid. Figure 4 shows the $0.5 \mu \mathrm{m}$ peak-to-peak orbital modulation for one day of data. Given AIRS's spectral sampling of 1/1200, the resulting day/night spectral calibration shift is about $4 \mathrm{ppm}$.

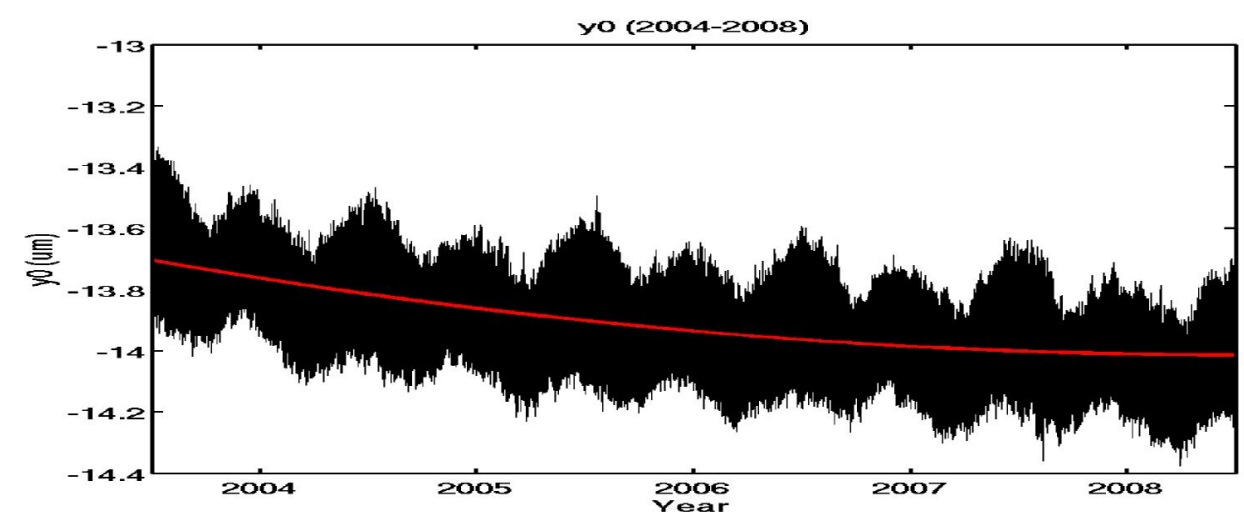

Figure 5. Changes in 5 years of AIRS data in terms of $y_{0}$ as a function of time. The red line shows the long-term component: an annual trend of $-0.07 \mu \mathrm{m}$ per year early in the mission, leveling off around 2008.

5 has 10 peaks related to the sun crossing the equator twice per year. The long-term trend (red line) may be linked to relaxation processes in the spacecraft or to the solar cycle. The peak-to-peak modulation of the focal pane position for the entire data period is less than one micron, equivalent to an $8 \mathrm{ppm}$ shift in the frequencies. For data assimilation for weather forecasting this shift can be ignored. This is the principal reason why the AIRS data were distributed for the past six years to the weather forecast centers as a Level-1B radiance product, i.e. without an explicit frequency recalibration.

If the long-term trend is removed, the envelope of the $\mathrm{y}_{0}$ is defined by a $0.4 \mu \mathrm{m}$ peak-to-peak orbital cycle, with a $0.2 \mu \mathrm{m}$ peak-to-peak seasonal modulation superimposed. The largest possible difference between two observations at the extremes of long-term, orbital, and seasonal cycles is less than $1.0 \mu \mathrm{m}$ or $8 \mathrm{ppm}$. 


\subsection{Approaches to spectral shifts}

For applications such as temperature and moisture retrievals in support of weather forecasting an 8 ppm uncertainty or even an $8 \mathrm{ppm}$ day/night or long term shift is unimportant, since the measurement accuracy from any one spectrum is dominated by random noise. Since the spectral shift observed in the AIRS data is very small, our approach for the past six years has been to ignore it. Researchers in need of higher spectral fidelity were able to minimize the impact of spectral uncertainty by avoiding use of specific channels at high gradients in the spectrum or by calculating the radiance for the SRF position as a function of time. But random noise cancels when large amounts of data are averaged for climate applications, so the interpretation of the data for climate is limited by uncertainties in the absolute radiometric and spectral calibration. Spectral calibration errors are likely to have complicated non-Gaussian and non-stationary characteristics, including day/night, clear/cloudy, and seasonal components. These climate applications may require an explicit correction for spectral shifts.

The most conservative approach to spectral shifting is to provide the users with an accurate assessment of time-varying frequencies but to leave the measured radiances unaltered. This method puts a burden on users to compensate for shifts as they use the data. AIRS v6 will support this approach with an improved assessment of time-dependent frequencies in the Level-1B products. Section 2.1 below details several approaches to identifying time-dependent frequencies, including the approach used in the upcoming version 6 of AIRS Level-1B products.

In order to make the AIRS data easier to use, a new Level-1C product is introduced in which radiances are shifted to a static nominal frequency set. This approach is very convenient for users, who can simply start using Level-1C where they previously used Level-1B. While minor artifacts from the shifting process make this approach slightly less accurate than using the time-dependent frequencies, it is still much more accurate than to ignore the shifts. Section 2.2 below explains how the shifting is accomplished and shows the accuracy attained.

\section{AIRS V6 SPECTRAL CALIBRATION}

The spectral calibration of AIRS has two components.

1) Determine the frequencies of the SRF centroids at the time of the observation.

2) Shift the observed radiances from the true (but time dependent) frequencies to a fixed set of frequencies.

\subsection{Determining the SRF frequencies.}

The AIRS Level-1B software from the start has determined the AIRS frequency using clear upwelling atmospheric spectra $^{5}$. These frequencies were written in the Level-1B product but were otherwise ignored. The determination of the spectral frequencies relies on the fact that spectral absorption and emission lines for water vapor, $\mathrm{CO}_{2}$, and other gasses always occur at precisely the same frequencies. An observed spectrum can be correlated to calculated spectra at a variety of shifts. The atmospheric state used in these correlations need not be perfect because the correlation process naturally ignores any bias or scaling difference. Differences in mean temperature will cause a bias between observed and calculated spectra, and differences in lapse rate cause scaling differences, but the fixed frequencies of spectral features guarantee that the correlation will be highest at the true shift. Because the AIRS detectors are rigidly mounted on the focal plane, the grating model equation and the shift $\mathrm{y}_{0}$ measured at any point in the spectrum can be used to calculate the frequencies of all channels.

The small size of the radiometric changes from spectral shifting relative to the instrument's background noise makes the measurement of $y_{0}$ quite challenging. Care must be taken first in the selection of the spectral regions in which the measurement will be made and also in the details of selecting and combining individual noisy observed spectra to get an accurate result.

The first consideration in choosing the spectral region is whether spectral lines or manifolds of lines can be observed with sufficient spectral resolution. The spectral calibration is SI traceable if the spectral resolution eliminates pseudo 
spectral shifts due to blending of $\mathrm{CO}_{2}$ and water vapor lines. This empirically requires a spectral resolution of about $1 / 2$ $\mathrm{cm}^{-1}$ or better. AIRS's resolution of $1 / 1200$ meets this standard at the longwave end, for frequencies under $\sim 750 \mathrm{~cm}^{-1}$, and so this region is favored for AIRS spectral calibration. The primary spectral feature selected for AIRS is the $\mathrm{CO}_{2}$ p-branch region between 728 and $780 \mathrm{~cm}^{-1}$ shown in Figure 6. Results from this region and the water band between 1400 and $1440 \mathrm{~cm}^{-1}$ agree very well. It is interesting to note that the operational IASI spectral calibration uses the resolved $\mathrm{CO}_{2}$ absorption lines between 2340 and $2380 \mathrm{~cm}^{-1}$ based on similar considerations.

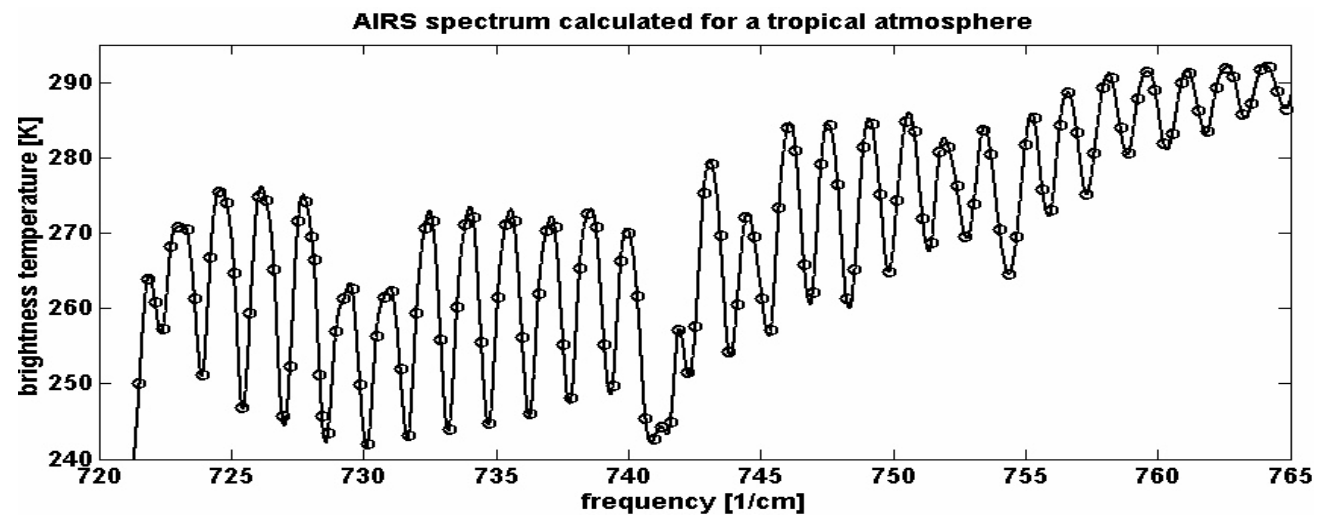

Figure 6. The primary spectral region used in AIRS spectral calibration
As shown in the AIRS Level 1B ATBD, the accuracy of the spectral calibration using upwelling radiances is proportional to the noise-equivalentdelta-temperature (NEdT), in units of $\mathrm{K}$, i.e. the smaller the better, and inversely proportional to the gradient between spectral features (the

steeper the better) and the square-root of the number of resolved spectral features (the more the better).

AIRS uses two related methods to measure $\mathrm{y}_{0}$ for each 6-minute granule. Section 2.1 describes an off-line approach using Level-2 cloud-cleared spectra; section 2.2 describes an approach using Level-1B spectra. We then fit models to the measurements to produce noise-reduced estimates of spectral shifts in the past and robust predictions of future shifts.

Using Level-2 cloud-cleared spectra ${ }^{6}$ minimizes the risk of using spectra that are contaminated with clouds. This method also has the advantage that a retrieved atmospheric state is available and so a spectrum can be calculated which closely matches current conditions. Because this method depends on Level-2 output, however, it cannot provide frequency information to the Level-2 process. There is a risk that systematic errors in Level-2 cloud cleared spectra and retrieved atmospheric state could lead to systematic errors in derived shift, but excellent agreement between results in different spectral bands strongly suggests that any such errors are small. The Level-1B method has to avoid clouds and must work without precise knowledge of the current atmospheric state.

\subsubsection{Spectral calibration using cloud-cleared radiances}

This method retrieves a granule-average $\mathrm{y}_{0}$ from the AIRS Level-2 cloud-cleared radiance and support products. The AIRS Level-2 support product provides a high vertical-resolution retrieved atmospheric state, suitable for direct use in the AIRS fast forward model ${ }^{7}$. The cloud-cleared radiances provide one spectrum for each of the 1350 Fields of Regard (FORs) in each six-minute granule. This spectrum has been derived in the Level-2 retrieval process by combining the spectra from 9 underlying raw Level-1B Fields of View (FOVs). For cloudless scenes and for channels whose atmospheric weighting function is completely above any clouds present, the cloud-cleared spectra are averages of the raw spectra. Everywhere else, the spectra are linear combinations of raw spectra calculated to remove the effects of clouds.

First we subset the spectra according to the internal quality flags, retaining only FORs with good cloud clearing quality and good temperature profile quality from the top of the atmosphere to the lower troposphere. We then convert observed cloud-cleared radiances for a pair of channels on top of strong absorbing lines, and another pair of channels between such lines, into Brightness Temperatures (BTs). We define the BT difference of these two pairs as the "spectral contrast" of the FOR, and retain only spectra with spectral contrast greater than the median for that granule. 
With the FOR selection now complete, we calculate radiances for the channels in our spectral feature using the AIRS fast model and the retrieved atmospheric state contained in the Level-2 profiles. We do this for two different $\mathrm{y}_{0} \mathrm{~s}$ separated by $2 \mu \mathrm{m}$ and centered near where we expect to find the actual $\mathrm{y}_{0}$. The observed and calculated radiances are each then averaged over the FORs to generate granule-mean spectra. The two calculated spectra are linearly interpolated in $\mathrm{y}_{0}$ onto a $0.02 \mu \mathrm{m}$ spaced grid spanning the range between the two $y_{0}$ s. Finally, we calculate the correlation coefficient between the cloud-cleared spectra and every grid point in our interpolated calculated spectra. The retrieved $\mathrm{y}_{0}$ is the one with the largest correlation.

\subsubsection{Real-time spectral calibration}

This method calculates a granule-average $\mathrm{y}_{0}$ from the AIRS Level-1B Radiances. The Level-1B radiances provide one spectrum for each of the 12150 Level-1B FOVs.

While the Level-2 method uses retrieved atmospheric states to dynamically calculate predicted spectra, this method instead relies on a library of representative precalculated spectra, each simulated over a range of scan angles, surface elevations, $\mathrm{CO}_{2}$ levels, and $\mathrm{y}_{0}$ shifts. The library is first interpolated to the nominal $\mathrm{CO}_{2}$ and elevation, and all spectra corresponding to atmospheric states outside the expected range for the current combination of month and latitude are excluded.

Spectra are accumulated separately for each of 5 scan angle ranges and up 50 spectral features. Only spectral features that show a high correlation ( 0.995 in prototype system) with at least one of the reference spectra are retained. This excludes cloudy cases and others with unexpected atmospheric states. After included spectra are selected, a multivote scheme is used to select the reference spectrum which best satisfies the largest number of individual spectra. Spectra are again compared with just that single reference spectra, and those with low correlations $(<0.995)$ are rejected. Surviving spectra are then combined across the granule to get a single granule-mean spectrum, for each scan angle bin. Correlations are then calculated between the combined observed spectrum and the selected reference spectrum at 5 shifts: $\{-12 \mu \mathrm{m}$, $-13 \mu \mathrm{m},-14 \mu \mathrm{m},-15 \mu \mathrm{m},-16 \mu \mathrm{m}\}$. Fitting a parabola through the correlations at the 5 shifts and taking the shift at which the parabola has its maximum gives the retrieved $\mathrm{y}_{0}$ shift. The measured shifts for all features and scan angle bins are combined to give a single value for the granule.

Throughout the process, several quality checks are performed, such as ensuring enough curvature on the parabola to get a reliable peak, checking the fitted correlation against a higher threshold, and rejecting extrapolated shifts. The results were tested on simulated data at different known shifts. The RMS error compared to the simulated shift was less than 0.2 $\mu \mathrm{m}$ in the prototype software.

\subsubsection{A model of temporal variation of AIRS $y_{0}$}

The off-line method of measuring $\mathrm{y}_{0}$ from cloud-cleared radiances described in section 2.1.1 was applied to 5 years of AIRS data. First a fit was used to discover and remove the long-term trend. The red line shown in Figure 5 above is that long-term trend. Figures 4 and 5 show that $\mathrm{y}_{0}$ varies in a very periodic fashion over a wide range of timescales. FFT (Fast Fourier Transform) analysis of $y_{0}$ shows clear cycles with periods of 1 orbit, 1 day, $1 / 2$ year, and 1 year, plus harmonics of these periods as expected from the varying heat load on the spacecraft described in section 1.2.

The smoothed $\mathrm{y}_{0}$ shown in Figure 4 was obtained by filtering out lower-amplitude terms and all terms corresponding to frequencies higher than 80 cycles/day. The largest differences between the raw and fit data occur in the high latitudes due to low spectral contrast in these relatively isothermal cases and to the sensitivity of the calculation to spectral contrast. The standard deviation of the differences between the data and fit gives the accuracy $0.05 \mu \mathrm{m}$ with worst-case disagreement of only $1.5 \mu \mathrm{m}$. Most of this is probably measurement noise.

The $\mathrm{y}_{0}$ values and derived frequencies from this model will be output by Level-1B, although the raw output of the Level1B method will also be output. The model has the advantage over the other methods in that it reduces measurement noise and can always return a value for every six-minute granule. Because it is a smooth model of cyclical behavior it can also 
provide shift information at a very fine temporal resolution: for every 2.67 -second instrument scan instead of just once per 6-minute granule. Level-1C will shift the observed spectrum from the current channel frequencies to the static frequency set.

When version 6 (v6) of AIRS science software is released, the model will be used to reprocess data from the start of mission in 2002 up to the present day. The model will also be used in near-real-time processing of current data until it is replaced by v7 approximately 2 years later. For all data up to the time at which the model is finalized it will be reproducing measured shifts but with measurement noise reduced by the fitting process. For later data, the model will be projected forward. The model may need to be updated during those 2 years and reprocessed back to the time at which it became inaccurate. Thus weather-quality Level-1B and Level-1C products will always be available rapidly and continuous climate-quality products will usually be available within days of observation but always within 1 month.

\subsubsection{Application of shifts measured in one frequency band to other bands}

Because the measurement of $y_{0}$ primarily uses module M-10, the absolute frequency uncertainty of that module is limited only by measurement uncertainties. For all other modules there is an additional transfer uncertainty due to uncertainty in the precise relative positions of the detector modules on the focal plane, summarized in Table 1 . The $3^{\text {rd }}$ column of Table 1 shows results from a preliminary attempt to measure $\mathrm{y}_{0}$ independently on each module using a small subset of AIRS data. Since no $\mathrm{y}_{0}$ solution was found for M-06 and M-02a in orbit, prelaunch $\mathrm{y}_{0}$ values are used.

Worst-case uncertainties are used for these modules in order to allow for possible changes between the pre-launch and onorbit behavior. It is anticipated that more thorough future tests will decrease these uncertainties substantially. The $4^{\text {th }}$ column gives the RMS brightness temperature change over all channels in a module for a simulated $1 \mathrm{ppm}$ shift. The $5^{\text {th }}$ column multiplies the previous two columns to yield an assessment of the uncertainty in units of temperature.

Generally the uncertainty in the position of a module is related directly to the requirement for the frequency knowledge of that module, so higher errors go with modules with lower knowledge requirements. Note that although the offsets between modules are not known precisely, they are know to be fixed -- the rigid nature of the AIRS focal plane ensures that changes in $\mathrm{y}_{0}$ measured in one frequency band are applicable to all bands. A full exploration of this topic is beyond the scope of this paper.

\subsection{Shifting the radiances from the true frequencies to a fixed frequency set.}

In the NASA EOS terminology the Level-1B product consists of the time/position tagged radiances at the spectral positions of the SRF. The positions of the SRF at the time of the observation are specified in the data record (using methods 2 and 3). The radiative transfer calculations required to convert the observed radiances to geophysical quantities are then carried out using the SRF frequencies at the time of the observation. Since this may be too involved for some 
investigators, a Level-1C product is also generated, which contains the radiances shifted to a fixed frequency grid. Nominal AIRS frequencies were agreed to at the start of routine data flow in September 2002 and correspond to a $y_{0}$ of $-13.5 \mu \mathrm{m}$.

In the following we describe the approach to shifting AIRS Level-1B data from the SRF centroids at the time of the observation to the Level-1C fixed frequency grid.

The frequency shift from the SRF centroids to the fixed frequency grid (nominal frequency) is a small correction, never exceeding $8 \mathrm{ppm}$, which is $2 \%$ of the space between adjacent channels. To make this small correction we need only an estimate of the local slope of radiance as a function of frequency. We use a cubic spline to interpolate the spectra onto the new frequency grid.

One complication to using a cubic spline is that it requires values for radiances of neighboring channels, and in some cases those neighbors are not of sufficient quality. So we first fill lower quality channels: those with a noise level over $1.0 \mathrm{~K}$ and those that are flagged bad by offline or upstream processes. This process applies to about 100 of 2378 channels. Channel filling is a two-stage process. First a rough fill is made either using the value of a single, well-matched neighboring channel or a value interpolated between the values of two such neighbors. Then the spectrum is passed through a principal components process and a new value is calculated.

Another challenge to the spline approach is the fact that the AIRS spectrum is slightly undersampled. This leads the cubic spline to systematically underestimate the adjustment needed in some channels and to systematically overestimate others. We use the following algorithm to modify slope at each channel from the cubic spline fit.

In formula (1), $R_{0}$ and $R$ represent the spectrum before and after the resampling, and $v$ is frequency, $\frac{d R}{d v}$ is the true slope of the spectrum.

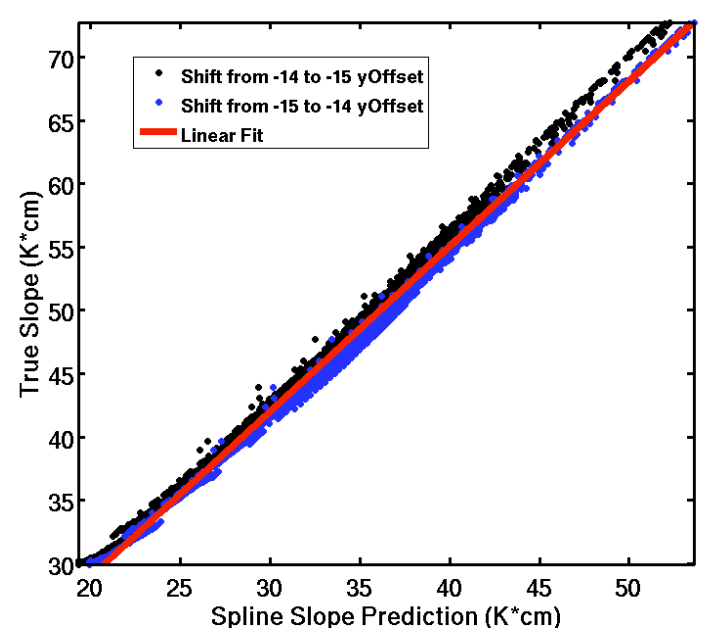

Figure 7. Correlation between the true simulated slope and spline fit slope for AIRS channel \#100 at $674 \mathrm{~cm}^{-1}$. Black dots represent a shift from $-14 \mu \mathrm{m}$ to $-15 \mu \mathrm{m} \mathrm{y}_{0}$, and the blue dots are the opposite. The red line is the linear fit. For this channel the spline consistently underpredicts the shift needed.

$$
R=R_{0}+\frac{d R}{d v} \cdot \Delta v
$$

The true slope of the spectrum is related to the slope from cubic spline fit by formula (2), where $\frac{d R^{S}}{d v}$ is the slope from cubic spline fit, and $A$ and $B$ are two frequency- or channel-dependent parameters derived from simulated results as shown below.

$$
\frac{d R}{d v}=A \frac{d R^{S}}{d v}+B
$$

Finally we calculate the resampled spectrum by formula (3),

$$
R=R_{0}+\left(A \frac{d R^{S}}{d v}+B\right) \cdot \Delta v
$$

We use one day of simulated spectra on December 8, 2008 to calculate channel-dependent parameters $A$ and $B$. These spectra include both clear and cloudy observations at $\mathrm{y}_{0}$ values of $-14 \mu \mathrm{m}$ and $-15 \mu \mathrm{m}$. Figure 7 shows the sample correlation between the simulated slopes (truth) and the slopes from cubic spline fit for channel $\# 100\left(674 \mathrm{~cm}^{-1}\right)$. The $A$ 
and $B$ parameters are then calculated from a linear fit. The resampled spectra from formula (3) are then compared to the simulated spectra (truth) in order to calculate the residual bias shown in Figures 8-11.

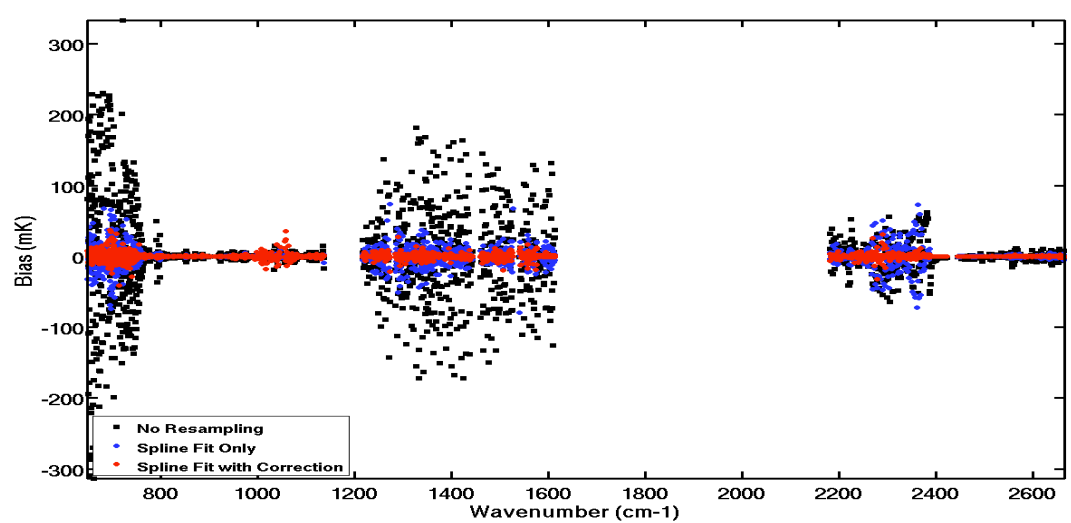

Figure 8 . The biases in one granule: the black dots are the differences between simulated $-14 \mu \mathrm{m} \mathrm{y}_{0}$ and $-15 \mu \mathrm{m} \mathrm{y}_{0}$ representing the error induced by using data with an uncorrected $1 \mu \mathrm{m}$ shift; the blue dots are the differences between data simulated at $-14 \mu \mathrm{m} \mathrm{y}_{0}$ and then converted using cubic spline resampling to $-15 \mu \mathrm{m}$ $\mathrm{y}_{0}$ and data simulated at $-15 \mu \mathrm{m} \mathrm{y}_{0}$; and the red dots show the modified spline fit compared to the truth.

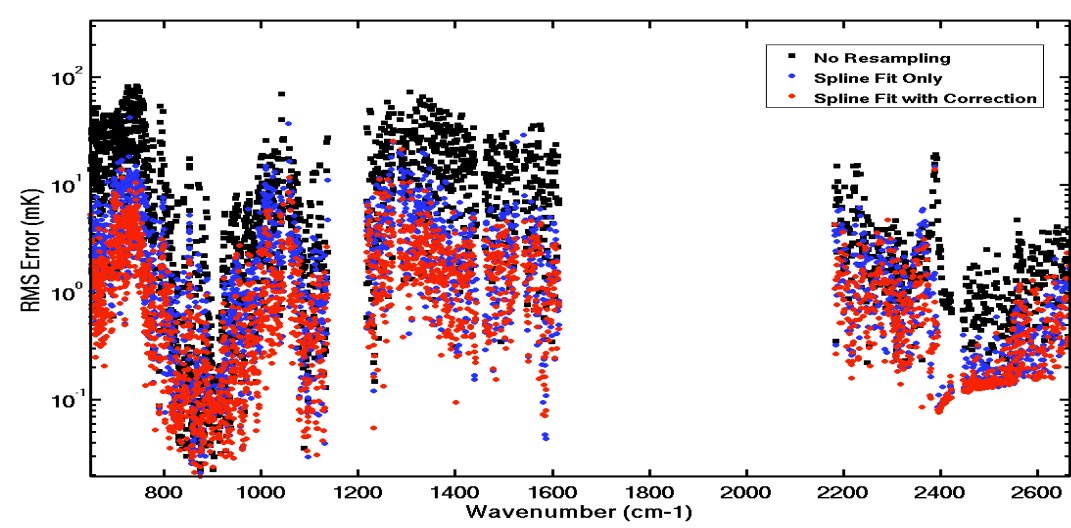

Figure 10. Same as Figure 8 except that RMS replaces bias

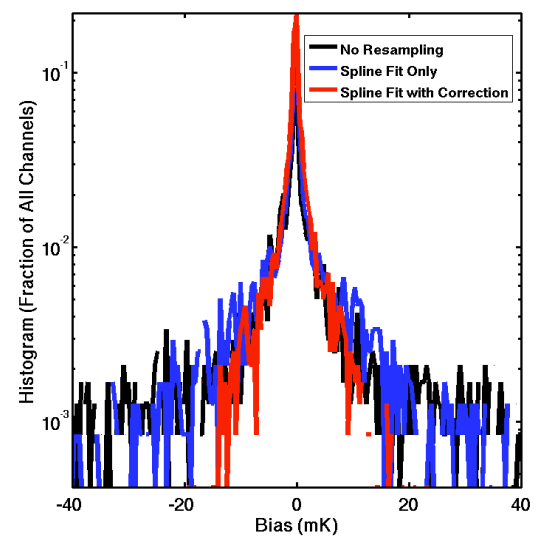

Figure 9. A histogram of the data shown in Figure 8.

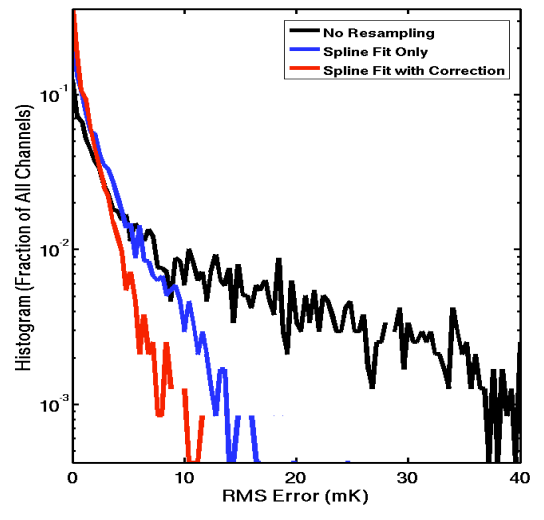

Figure 11. A histogram of the data shown in Figure 10.

Figures 8 and 9 show how much the bias over one sample granule improves from no correction (black dots) to spline fit (blue dots) to spline plus correction (red dots). The biases decrease from $\pm 300 \mathrm{mK}$ to within $\pm 10 \mathrm{mK}$.

The RMS (Root Mean Square) of the differences is plotted in Figure 10. It shows that the resampled accuracy is always better than $12 \mathrm{mK}$. In fact, the RMS over all channels is only $1.3 \mathrm{mK}$.

\subsection{Residual errors}

What are the practical implications of these spectral errors and corrections? We return to the three options

\begin{tabular}{|c|c|c|}
\hline Approach & Typical Error & Worst Case Error \\
\hline Ignore & $45 \mathrm{mK}$ & $480 \mathrm{mK}$ \\
\hline Adjust to static set (Level-1C) & $4.7 \mathrm{mK}$ & $110 \mathrm{mK}$ \\
\hline Use with knowledge of current shift & $4.5 \mathrm{mK}$ & $80 \mathrm{mK}$ \\
\hline
\end{tabular}

Table 2. Errors in brightness temperature attributable to AIRS frequency variation. 
discussed in section 1.3: ignoring the effect, using unmodified radiances with the time-dependent frequencies, and using corrected radiances. Table 2 summarizes the errors of each.

When frequency shift effects are ignored, i.e. uncorrected Level-1B radiances are used, an effective error is introduced into analyses that compare AIRS products from different times. This error depends critically on the specific set of frequencies and times used. A worst-case error using the highest relative shifts seen in Figure 5, $1.2 \mu \mathrm{m}$, and the highest spectral sensitivity seen for AIRS, $400 \mathrm{mK} / \mu \mathrm{m}$, gives a worst-case error of $480 \mathrm{mK}$. Typical values of $0.5 \mu \mathrm{m}$ shift and $90 \mathrm{mK} / \mu \mathrm{m}$ give a typical error of $45 \mathrm{mK}$.

The error when using unmodified radiances with time-dependent frequencies in the radiative transfer code is simply the error in measuring the $\mathrm{y}_{0}$ shift multiplied by the sensitivity of the radiances to $\mathrm{y}_{0}$. Worst-cases error in $\mathrm{y}_{0}$ is $0.2 \mu \mathrm{m}$, and the highest sensitivity is $400 \mathrm{mK} / \mu \mathrm{m}$, giving $80 \mathrm{mK}$ worst-case error. Typical error in $\mathrm{y}_{0}$ is $0.05 \mu \mathrm{m}$ and typical slope is $90 \mathrm{mK} / \mu \mathrm{m}$, so typical error is $4.5 \mathrm{mK}$.

When spectral shift is measured and corrected in the Level-1C product, then the residual error is a combination of the uncertainty in the measurement of the $\mathrm{y}_{0}$ shift above and the error introduced in the application of the shift. Worst-case error in shifting is $30 \mathrm{mK}$ (Figure 10), giving $110 \mathrm{mK}$ overall. Adding (RMS) the $1.3 \mathrm{mK}$ typical shifting error to the 4.5 $\mathrm{mK}$ error from section 2.3.2 gives a final typical error of $4.7 \mathrm{mK}$.

\section{CONCLUSION}

In order to use hyperspectral data for climate applications, the frequencies and shape of the spectral response function have to be known and extremely stable. We show that $0.5 \mathrm{ppm}$ knowledge is required in the SRF centroid positions in areas of high spectral contrast. Analysis of six years of AIRS data shows variation in the position of the SRF centroid up to $8 \mathrm{ppm}$, but methods are now in place to determine the SRF centroids to within $0.4 \mathrm{ppm}$. The residual errors are typically $5 \mathrm{mK}$ or less, far smaller than the noise level and is comparable to only one half year's expected climate change signal.

There are two spectral ranges to which the static offset from the regions used for shift determination is not well characterized because these regions have insufficient contrast at AIRS spectral resolution. The sensitivity of the radiometric signal from any hyperspectral instrument is very sensitive to the spectral calibration. Care must be taken in comparing AIRS data with data from other hyperspectral instruments.

\section{ACKNOWLEDGEMENTS}

The work described in this paper was carried out at the Jet Propulsion Laboratory, California Institute of Technology and at University of Maryland Baltimore County, under a contract with the National Aeronautics and Space Administration.

\section{REFERENCES}

[1] Aumann, H. H., Chahine, M. T., Gautier, C., Goldberg, M. D., Kalnay, E., McMillian, L. M., Revercomb, H., Rosenkranz, P. W., Smith, W. L., Staelin, D. H., Strow, L. L. and Susskind, J., "AIRS/AMSU/HSB on the Aqua Mission: Design, Science Objectives, Data Products, and Processing Systems," IEEE Trans. Geo Sci. and Remote Sensing 41(2), 253 (2003)

[2] Blumstein, D., Chalon, G., Carlier, T., Buil, C., Hebert, P., Maciaszek, T., Ponce, G., Phulpin, T., Tournier, B., Simeoni, D., Astruc, P., Clauss, A., "IASI instrument: Technical overview and measured performances," Proc. SPIE 5543, $196(2004)$

[3] Glumb, R. J., Jordan, D. C. and Mantica, P., "Development of the Crosstrack Infrared Sounder (CrIS) sensor design," Proc. SPIE 4486, 411-424 (2002) 
[4] Strow, L. L. and Hannon, S. E., "A 4-year zonal climatology of lower tropospheric CO2 derived from ocean-only Atmospheric Infrared Sounder observations," J. Geophys. Res., 113, (2008)

[5] Aumann, H., Gaiser, S., Ting, D. and Manning, E., "AIRS Level 1B Infrared Spectrometer Channels ATBD”, http://eospso.gsfc.nasa.gov/eos_homepage/for_scientists/atbd/docs/AIRS/AIRS_L1B_ATBD_Part_1.pdf (2000)

[6] Susskind, J., Barnet, C. D. and Blaisdell, J. M., "Retrieval of Atmospheric and Surface Parameters From AIRS/AMSU/HSB Data in the Presence of Clouds," IEEE Transactions on Geoscience and Remote Sensing 41(2), 390 (2003)

[7] Strow, L. L., Hannon, S. E., De Souza-Machado, S., Motteler, H. E. and Tobin, D., "An overview of the AIRS radiative transfer model," IEEE Transactions on Geoscience and Remote Sensing 41(2), 303 (2003) 